\title{
Special Issue on Robots for Future Societies: Evaluating Social Acceptance and Societal Impact of Robots
}

\author{
Astrid Weiss · Manfred Tscheligi
}

Accepted: 22 September 2010 / Published online: 16 October 2010

(C) Springer Science \& Business Media BV 2010

Human-Robot Interaction research enters new areas of interest, besides technical developments and engineering approaches. The interest in the roles robots can possibly play in future societies steadily grows. Topics like acceptability, ethics, and sustainability become more and more spotlighted when studying social robots. Questions arise like: what makes a robot socially accepted (long-term), what are the societal implications of integrating robots into education, working or health contexts etc. All these topics will be addressed in this special issue. In this special issue, topical research will be presented on how socially acceptable robotic systems should be designed, developed, and assessed and what societal impact robots could have in the future. A combination of reviews on existing measures and metrics for the assessment of social acceptance, structural frameworks towards social and societal acceptance, and endings from Human-Robot Interaction studies will be discussed. Moreover, key challenges (and possible ways to address some of them) social robots pose in terms of technological, designorientated, and user-centered aspects will be highlighted. All articles in this special issue underline the importance of taking the aspect of social acceptability in social robotics research into account and place emphasis on how to integrate social acceptability into research projects.

In the first article "Review of human studies methods in HRI and recommendations", Cindy L. Bethel and Robin R. Murphy present a thorough survey on research methods for

\footnotetext{
A. Weiss $(\bowtie) \cdot M$. Tscheligi

HCI\&Usability Unit, ICT\&S Center, University of Salzburg,

Sigmund-Haffner-Gasse 18, 5020 Salzburg, Austria

e-mail: astrid.weiss@ sbg.ac.at

M. Tscheligi

e-mail: manfred.tscheligi@sbg.ac.at
}

human studies in social robotics. They describe the application of these methods with its advantages and disadvantages in detail and underline the challenges in human studies in HRI. Based on case studies they give recommendations for other researchers on how to generate valid data in human studies with social robots.

The second article "Assessing acceptance of assistive social agent technology by older adults: the Almere model", written by Marcel Heerink, Ben Kröse, Vanessa Evers and Bob Wielinga, presents a statistically validated acceptance model especially suited for evaluating interaction of elderly users with socially interactive systems. It is based on the Technology Acceptance Model (TAM) and was extended by relevant factors, which were identified in several user studies with elderly participants. Therefore, the Almere model offers other researchers theoretical and methodological grounding for HRI studies with elderly participants.

The next article "Expressing emotions with the social robot Probo", written by Jelle Saldien, Kristof Goris, Bram Vanderborght, Johan Vanderfaeillie, and Dirk Lefeber, describes the development of the socially interactive robot Probo. Probo is a huggable animal-like robot, especially designed to suit the needs of children. The article describes the robot's abilities to show emotional states based on Russel's circumplex model of affect and discusses the results of several user studies on the recognition of the underlying emotions. Thereby, the article shows how affective design can improve the social acceptability of a robotic platform.

Similarly, the article of Karla Conn Welch, Uttama Lahiri, Nilanjan Sarkar, and Zachary Warren, entitled "An approach to the design of socially acceptable robots for children with autism" presents how experiments with virtual social robots can contribute towards the development of future social robots for children suffering from Autism Spectrum Disorders (ASD). Therefore, the authors propose a virtual 
environment system for social interaction (VESSI) that can inform the design of robots in a very early stage of the design process to foster social acceptability.

Child-Robot Interaction is also the topic of the article from Tamie Salter, François Michaud, and Hélène Larouche, entitled "How wild is the wild? A taxonomy to characterize the wildness of Child-Robot Interaction". Based on a literature review combined with several user studies, the authors propose a framework of the degree of "wildness" in HRI studies with children. This framework should increase the standardization and comparability of user studies with children and thus help to identify key influence factors on (longterm) acceptability.

Similarly, in the article "Domestic Robot Ecology: An initial framework to unpack long term acceptance of robots at home" Sung, Grinter, and Christensen are investigating long-term acceptability "in the wild". The authors propose a framework for the home as human- robot interaction context, derived from the data of a long-term field study with roomba vacuuming robots in 30 households. The authors provide other researchers with implications for long-term interaction design in the domestic context.

Otherwise, Kristian Wasen is interested in how robots influence the working context. In his article "Replacement of highly educated surgical assistants by robot technology: A paradigm shift in the service sector", he presents the insights gained from ethnographic work place studies in the healthcare sector. His research revealed that surgical assistant robots are on the one hand well-adopted and accepted among operating surgeons. On the other hand a paradigm shift in society can be observed, namely that highly educated knowledge workers become replaced by robot technology.

The influence of robotic technology on a societal level is also discussed in the article "Robots in society, society in robots: Mutual shaping of society and technology as a framework for social robot design". The author, Selma Šabanović, presents a framework for developing and evalu- ating and evaluating robotic systems based on mutual shaping of societal needs and technological possibilities. Based on data collected through participant observation and interviews with Japanese and US roboticists, Šabanović demonstrates the technologically determinist notions of the dynamics between society and technology. To overcome this aspect, a valuable interdisciplinary approach for the integration of participatory design into social robotics research is presented.

The article of Pericle Salvini, Cecilia Laschi, and Paolo Dario on "Design for acceptability: The role of human factor in designing service robots" closes the special issue. The authors aim at broadening the view on 'acceptability' within the design of service robots. They propose an approach in accordance to the notion of Vicente's "Human-Tech Ladder", meaning that also ethical, legal, and societal concerns need to be taken into account. A key concept of this notion is "emergent property"-aspects of acceptability that only come into existences through the "situated" interaction of a user and a robot.

The idea for this special issue on "Robots for future societies: Evaluating social acceptance and societal impact of robots" evolved from the organization of a workshop on "Robots as Social Actors: Evaluating Social Acceptance and Societal Impact of Robotic Agents" held at IEEE RO-MAN 2008 and a follow- up workshop on "Societal Impact: How Socially Accepted Robots Can be Integrated in our Society" held at ACM/IEEE HRI 2009. Therefore, many thanks go to Professor Aude Billard and Professor Kerstin Dautenhahn, who were both actively involved in the organization of these workshops. Special thanks go to Professor Shuzhi Sam Ge, who invited us as guest editors and to the field experts who supported us in the reviewing process, who provided fruitful comments for improving the articles included in this issue. Finally, the editors hope that the readers will enjoy the individual articles and that they will inspire future research on social robotics. 